\title{
La evaluación continua en el Grado de Trabajo Social de la Universidad de Barcelona
}

\section{The continuous assessment on the degree of Social Work at the University of Barcelona}

\author{
Violeta QUIROGA RAIMÚNDEZ \\ Universidad de Barcelona \\ violetaquiroga@ub.edu \\ Ester MEna García \\ Fundación Pequeño Deseo \\ estermen@hotmail.com \\ Jessica PORLAN JuY \\ Patronat Comarcal de Serveis a la Persona del Baix Llobregat \\ jessicaporlanjuy@gmail.com \\ Esther MORALES MaRTíNeZ \\ Universidad de Barcelona \\ esther_molins@yahoo.es \\ Lidia MÁRQUEZ SÁNCHEZ \\ Universidad de Barcelona \\ marquezlidia@hotmail.com
}

Recibido: 19/03/2014

Revisado: 24/07/2014

Aceptado: 24/10/2014

Disponible on line: 10/12/2014

\begin{abstract}
Resumen
Las directrices del Plan Bolonia presentan un desafío importante en los sistemas educativos miembros de la UE porque conllevan un cambio radical en la forma de organizar el proceso educativo universitario. No obstante, cada estado posee una legislación que atiende a unos valores culturales, e idiosincrasia que, en ocasiones, pueden presentar divergencias en este proceso de unificación de criterios. Algunos países, entre ellos España, han aprovechado este contexto para replantearse la metodología docente.

El objetivo general de la investigación fue evaluar la implementación de la evaluación continua en el Grado de Trabajo Social de la Universidad de Barcelona para mejorar el conocimiento sobre la evaluación continua y detectar las necesidades y carencias que se estaban produciendo con la implantación de este nuevo enfoque, así como su impacto tanto en profesores como en el alumnado.

El trabajo de campo se realizó en la Facultad de Pedagogía de la UB sobre el grupo de estudiantes que estaba cursando el grado de Trabajo Social, los docentes que lo impartían, cargos de gestión de la Facultad de Pedagogía, además de expertos en la temática y otros referentes que aportaron sus experiencias en otras facultades. La metodología utilizada fue de carácter cualitativo y cuantitativo a través de entrevistas, grupos de discusión y encuestas al $80 \%$ de los alumnos que cursaban $4^{\circ}$ del Grado de Trabajo Social.

Después de poder contrastar las dos visiones principales que abarcaba esta investigación: alumnado y profesorado, pudimos destacar la falta de unidad de criterios en relación a lo que es la evaluación continua y cómo se debía aplicar y constatamos que otros factores incidían como elementos poco favorecedores para permitir un seguimiento y un retorno por parte del profesorado.

No obstante, a pesar de las deficiencias de la implementación de la evaluación continua en el grado de Trabajo social, los alumnos percibían que podrían aplicar los aprendizajes y conocimientos adquiridos una vez finalizaran el grado. Así podemos constatar que las deficiencias se ciñen más a la metodología de implementación que en los contenidos.
\end{abstract}

Palabras clave: evaluación continua, Plan Bolonia, aprendizaje, capacidades, competencias y habilidades, universidad, profesores, estudiantes, planes docentes. 


\begin{abstract}
The Bologna process has meant important challenges to national higher education systems in Europe that have had to modify the ways of organizing teaching. Each country has its idiosyncrasies and its cultural values embedded in their educational regulations that may mean that reforms move in different directions. Spain, like some other countries, has attempted to reform its teaching methods in the context of the Bologna process.

Our research has focused on the evaluation of how continuous assessment of students' performance has been implemented in the Degree in Social Work at the University of Barcelona. Our aim has been to know better how this process is being carried out and identify problems arising from its implementation, as well as its impact both on faculty and on students. Data was collected at the Facultat de Pedagogia of the UB from students, faculty and managers with direct relation to the degree in Social Work, and from experts from other areas of the university. We've used both qualitative and quantitative techniques, mainly through interviews, focus groups and a survey answered by 80 percent of final year students. After bringing together the points of view of students and faculty, the lack of a common definition and of common criteria on what is continuous assessment and how to put it in practice was apparent, and that some other factors were hindering follow-up and feedback to students.

Nevertheless, and despite the limitations in the implementation of continuous assessment, students think they will be able to use what they've learnt after graduating, and this suggests that the problems affect the implementation method rather than its contents.

Keywords: continuous assessment, Bologna process, learning, skills and competences, university, faculty, students, teaching plans.

Referencia normalizada: Quiroga Raimúndez, V., Mena García, E., Porlan Juy, J., Morales Martínez, E. y Márquez Sánchez, L. (2014): «La evaluación continua en el Grado de Trabajo Social de la Universidad de Barcelona». Cuadernos de Trabajo Social, 27(2): 353-363.
\end{abstract}

Sumario: Introducción. 1. Metodología. 2. Resultados de la investigación. 3. Conclusión. 4. Referencias bibliográficas.

\section{Introducción}

Las directrices del Plan Bolonia, presentan un desafío importante en los sistemas educativos miembros de la UE porque conllevan un cambio radical en la forma de organizar el proceso educativo universitario, no se debe obviar, en este punto, que cada estado posee una legislación que atiende a unos valores culturales, e idiosincrasia que, en ocasiones, pueden presentar divergencias en este proceso de unificación de criterios, es por este motivo que nos planteamos si el cambio de enfoque basado en la «enseñanza» a un nuevo modelo que incide en el «aprendizaje» está surgiendo el efecto deseado.

El objetivo general de este proyecto se ha centrado en evaluar la implementación de la evaluación continua en el grado de Trabajo Social de la Universidad de Barcelona, para, a partir de su análisis, poder contribuir en una mejora en la calidad de la enseñanza y formación de los futuros trabajadores sociales.

Esta investigación se realizó en la asignatura de Investigación Aplicada en Trabajo Social que se cursa en tercero del grado, en el marco de El Aprendizaje Servicio (APS de la Facultad de Pedagogía de la UB) en colaboración con el Departamento de Trabajo Social y Servicios Sociales de la Universidad de Barcelona, y se llevó a cabo de septiembre de 2012 a enero de $2013^{1}$.

Resultaba fundamental conocer las dos perspectivas que confluyen en el proceso de aprendizaje, la de los docentes y la del alumnado y si las políticas aplicadas incidían en un avance hacia una evaluación continua de calidad o si por el contrario, las dificultades en este terreno estaban incidiendo en una praxis con muchas evidencias evaluativas, pero que se alejaban del objetivo primero que era el aprendizaje real del estudiante.

Después de efectuar el análisis exhaustivo y poder confrontar las dos visiones principales que abarcaba esta investigación: alumnado y profesorado, detectamos la falta de unidad de criterios en relación a lo que es la evaluación continua y su aplicación.

Corroboramos que las deficiencias se ceñían más a la metodología de implementación que no

${ }^{1}$ En esta investigación han participado además de las autoras del artículo, 8 investigadores más: Batet, Josep Lluis; Bertrán, Laura; Castel, Sara; Martínez, Pilar; Martínez, Cristal; Mateos, Nerea; Peña, Minssu; Trapero, Paz. 
a los contenidos, ya que la mayoría de estudiantes los evaluaron de forma satisfactoria.

\section{Metodología}

La investigación social que llevamos a cabo fue de finalidad básica y aplicada a partir de fuentes mixtas. La profundidad, de tipo descriptiva y explicativa, la amplitud micro sociológica y el alcance temporal, seccional y sincrónico.

La estrategia de integración metodológica fue la complementación, diferenciando los siguientes grupos de actores: expertos, cargos de gestión de la Universidad de Barcelona, docentes y estudiantes.

La metodología que se utilizó fue de carácter cualitativo y cuantitativo. En relación, a la cualitativa se realizaron 3 entrevistas exploratorias a expertos (un experto en metodología y dos docentes con conocimiento de otras experiencias universitarias ${ }^{2}$ ), 3 entrevistas en profundidad a cargos de gestión y docentes (decana de la Facultad de Pedagogía y directora y jefe de estudios del Grado de Trabajo Social), y 4 grupos de discusión. Los grupos de discusión fueron un grupo a docentes formado por siete profesores y tres grupos de alumnos de los tres últimos cursos formados por doce, once y diez alumnos. El criterio de selección para el grupo de discusión con los docentes, se basó en que fueran profesores coordinadores de las asignaturas de formación básica u obligatoria. Cada una de las muestras para la selección fue no probabilística e intencionada.

En relación a la metodología cuantitativa, se pasó un cuestionario a 136 alumnos, el $80 \%$ de alumnos matriculados en $4^{\text {a }}$ curso del Grado de Trabajo Social de la Universidad de Barcelona.

\section{Resultados de la investigación}

\subsection{Contextualización}

La construcción del Espacio Europeo de Educación Superior es un proceso de consenso que culminó con la Declaración de Bolonia en 1999 en base a los principios de calidad, movilidad, diversidad y competitividad, con un sistema de calificación y créditos equivalente, conocido como ECTS (European Credits Transfer System) fundamentado en unidades de medida académica para que el trabajo desarrollado por un estudiante en cualquiera de las universidades de los estados miembros fuera reconocible en cuanto a nivel, calidad y relevancia (Ministerio de Educación, Cultura y Deporte, 2003).

Asimismo se pretendía también un cambio de paradigma en relación al sistema tradicional que basaba los procesos de enseñanza-aprendizaje en términos de imputs dando relevancia a las horas dedicadas a la enseñanza, pasando a un nuevo enfoque centrado en los outputs que se expresa en términos de competencias y resultados de aprendizaje. Las carreras se tenían que diseñar en base a las competencias que los alumnos tendrían que adquirir y que debían venir definidas en los planes de estudios. De esta manera los objetivos de aprendizaje, o lo que es lo mismo, las competencias, lo que los alumnos deben adquirir en cada asignatura, es lo que puede ser comparable dentro del EEES. Según Imbernon y Medina (2008), este nuevo modelo de enseñanza, exigía un esfuerzo en la planificación de las sesiones formativas, una previsión de los recursos personales y materiales y una nueva distribución, organización y utilización de los espacios educativos. La planificación hace referencia al hecho de diseñar y concretar unas intenciones educativas y la manera de conseguirlo, es decir, las actividades que deben realizarse. De esta manera, las actividades son el elemento central de este proceso de enseñanza y aprendizaje por lo que hay que tener en cuenta dos tipos de actividades: la actividad del alumnado y la del profesorado.

Ciertamente se ha producido un cambio de paradigma a nivel educativo que pretende estimular el aprendizaje no sólo en el transcurso del alumno en su vida académica, sino más bien en

${ }^{2}$ De especial relevancia es la entrevista realizada al experto Joan Mateo, teórico y experto en evaluación continua, catedrático de la Facultad de Pedagogía, presidente del Consejo Superior de Evaluación del Sistema Educativo y Secretario de Políticas Educativas del Departament d'Ensenyament (en el momento de realizar la entrevista). Las entrevistas exploratorias se realizaron a Xavier Giménez, profesor de la Facultad de Química de la Universidad de Barcelona, quién nos aportó su visión y experiencia en otra facultad de la UB, y Tomasa Báñez, profesora del Grado de Trabajo Social de la UB, quien nos facilitó información sobre la implementación de la evaluación continua del Grado de Trabajo Social en la Universidad de Zaragoza, de donde ella procedía y había sido jefe de estudios en los últimos años. 
la formación constante a lo largo de su vida, sin embargo la repercusión del cambio paradigmático es poco apreciable debido al corto espacio de tiempo que ha transcurrido y que dificulta hacer valoraciones.

En España, al igual que en Italia o Francia, la tradición en la enseñanza proviene del modelo docentista, o lo que es lo mismo: clases magistrales con un elevado número de alumnos tomando notas son el eje principal sobre el que se basa el aprendizaje. Esto contrasta con otros modelos como el anglosajón o el estadunidense donde se realizan menos clases magistrales, con menor número de alumnos por grupo pero donde hay un seguimiento más individualizado de los estudiantes. El Plan Bolonia no proponía implantar la evaluación continua como modelo estándar aunque sí establecía centrar los aprendizajes en el estudiante. La renovación metodológica, a pesar de no ser un principio explícito en la declaración de Bolonia, de forma indirecta se ha entendido que es indispensable para llegar a una formación basada en competencias (García Martínez, 2009). En la implementación en España de Bolonia, uno de los elementos centrales sobre el que se ha construido la metodología para el cambio del paradigma enseñanza-aprendizaje ha sido la evaluación continua.

\subsection{Concepto de la evaluación continua}

El sistema de evaluación continua se apoya en una perspectiva constructivista de orientación sociocultural (Coll, Martín y Onrubia, 2001) donde la evaluación, la ayuda educativa y el aprendizaje están estrechamente relacionados. La evaluación, desde esta perspectiva está considerada como un instrumento fundamental para que el profesor pueda regular su acción docente y el alumno pueda regular su proceso de aprendizaje (Mauri y Rochera, 1997).

Desde esta nueva percepción desaparece la distinción entre «evaluación formativa y sumativa que quedan definitivamente subsumidas en el nuevo concepto de evaluación continua. Por evaluación continua no hay que entender que se debe estar continuamente evaluando sino que los procesos de aprendizaje y los de evaluación forman parte del mismo «continuum». (Escofet, Martínez y Mateo, 2009) y proporciona al profesor información que le permite intervenir para mejorar y reorientar el proceso de aprendizaje (López, 2001)
Delgado y Oliver (2006) afirman que la evaluación es continua y progresiva, ya que las actividades evaluables deben facilitar la asimilación y el desarrollo progresivo de los contenidos $\mathrm{y}$ de las competencias que deben alcanzarse. Mateo en la entrevista describe que la evaluación continua es el proceso de evaluación que se une simbióticamente al propio proceso del aprendizaje de los alumnos y va interaccionando con él todo el tiempo; lo hace de forma que cuando evaluamos aprendemos y cuando aprendemos debemos evaluar.

Podemos concluir que el eje central de la evaluación continua es el propio proceso de aprendizaje que a través de un feedback continuo entre profesor y alumno, posibilite la progresión, mejora e interiorización de los aprendizajes.

Sin embargo, en base a nuestra investigación, según las declaraciones en el grupo de discusión de docentes de Trabajo Social de la UB, quedó de manifiesto que el concepto de evaluación continua, conlleva ambigüedades y permite interpretaciones muy diversas. Los docentes apuntaban que había una gran diferencia entre lo que se decía de la evaluación continua en la teoría y lo que realmente se hacía en la práctica, que cada uno daba un valor diferente al concepto de la evaluación continua y que el hecho de que no todos entendieran lo mismo provocaba una gran confusión.

Ante el planteamiento de si existían criterios claros y definidos sobre qué es la evaluación continua, en las entrevistas a los cargos de gestión de Trabajo Social de la UB, el jefe de estudios del Grado de Trabajo Social y Servicios Sociales de la UB, considera que no hay un consenso generalizado y único entre el profesorado, ya que «a nivel teórico podemos estar todos de acuerdo en una misma definición pero a la hora de llevarlo a la práctica es cuando hay ciertas divergencias»». Contrariamente, la decana de la Facultad de Pedagogía de la UB, cree que sí que existen criterios definidos pero no niega que existen «inconsistencias que no están bien resueltas», por lo que considera que la supervisión es algo fundamental.

En los cuestionarios a los alumnos que estaban cursando $4^{\circ}$ curso de trabajo social, la respuesta fue contundente ya el 97 por ciento de los alumnos consideraba que no existía unidad de criterios entre los docentes sobre la evaluación continua. 


\subsection{Metodología. Enseñanza vs Aprendi- zaje}

El objetivo más importante en la metodología de la evaluación continua es Aprender a aprender (Escofet, Martínez y Mateo, 2009). Conseguir que la persona se apropie de su propia capacidad para seguir aprendiendo es uno de los mandatos más potentes de nuestra época. Es necesario dotar al alumno de la capacidad autónoma de aprender y mantener en él la motivación para seguir aprendiendo (Escofet, Martínez y Mateo, 2009). En relación a este concepto, Mateo, apuntaba que el objetivo más claro es desarrollar la autorregulación y la autonomía de los aprendizajes.

Si nos remitimos a los propósitos que se establecen dentro del Plan Bolonia, concebido como un cambio en el paradigma educativo, el proceso de aprendizaje consiste en la adquisición de unas competencias basadas en conocimientos, capacidades, habilidades y actitudes, las cuales deben constar en los planes docentes. Así pues, el plan docente debería ser la hoja de ruta para conseguir esos objetivos, sin embargo encontramos que las competencias de los planes docentes del grado de Trabajo Social eran genéricas y abstractas, e incluso en muchas ocasiones no concordaban con la realidad que se ofrecía en la asignatura.

La importancia de la adquisición de una competencia, se sitúa en el cómo se ha producido el aprendizaje de la misma y se pretende que el profesor integre el proceso evaluativo en el de aprendizaje y le confiera una mayor profundidad para que el estudiante gracias a la evaluación continua, tome consciencia de sus logros y de sus deficiencias.

El estudiante debe ser un sujeto activo en su propio proceso de aprendizaje incrementando la capacidad para gestionar de forma autónoma las competencias que deberá ir adquiriendo no sólo en su primera etapa de formación sino a lo largo de toda la vida.

Mateo como experto tiene claro que para ello la única forma actual que la gente conoce es gracias a la evaluación de lo que tú estás aprendiendo, tomando conciencia de lo qué sabes y de lo qué no sabes y sobretodo de lo qué sabes cómo lo has aprendido. La mayoría de los entrevistados, dan importancia a este concepto entendido como el aprendizaje a lo largo de toda la vida.
En cuanto a las metodologías apropiadas para favorecer este proceso de aprendizaje, Mateo nos habla de un cambio en el «paradigma» (...) En lugar de metodologías orientadas a cómo enseñar, son metodologías orientadas en cómo puede aprender el alumno. (...) Las metodologias modernas giran alrededor de los aprendizajes basados en problemas, los aprendizajes basados en actividades (...) El acompañamiento del alumno es una oportunidad de aprendizaje

\subsection{Evidencias}

En relación al proceso evaluativo, Mateo opina que las asignaturas no deberían programarse por temarios sino por actividades, y que estas sirviesen para que el alumno aprendiese a aprender, ayudado en su proceso por un feedback ágil con el profesor: Cada actividad es un conglomerado que permite al alumno integrar los conocimientos de forma aplicativa sobre el contexto de realidad y le obliga a buscar y a encontrar información, aplicarla y luego contrastarla con el profesor.

Así pues la cuestión más importante en este proceso, centra su objetivo en conseguir que las evidencias tengan unos objetivos claros en cuanto a su función de adquirir los conocimientos, habilidades y capacidades presentes en los planes docentes; pero aquí de nuevo existen incongruencias, ya que a pesar de que los expertos aseguraban que deberían ser pocas y con un sentido nuclear, los mismos docentes reconocían abiertamente que debería haber menos cantidad de evidencias exigidas, pero con más calidad.

En relación a la calidad de las evidencias, el 81 por ciento de los alumnos creía que la calidad de las evidencias era regular, deficiente o muy deficiente. En cuanto a la valoración de las evidencias, los alumnos daban un 5,7 en una escala de 1 a 10 , en relación a la utilidad de las evidencias como medio para adquirir los conocimientos, habilidades y capacidades que se proponen en los planes docentes

En los grupos de discusión, los alumnos manifestaban abiertamente y de forma reiterada el malestar que les provocaba sentir que son sobrecargados con multitud de entregas de evidencias, muchas de ellas sin sentido aparente con los objetivos de la asignatura, con temáticas repetidas, y la mayoría de ocasiones, sin posibilidad de retorno por parte del profesorado. 


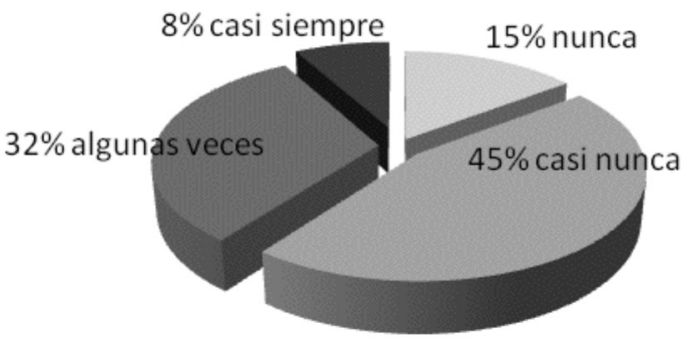

Nunca

Casi Nunca

- Algunas veces

Casi siempre

Gráfico 1. Retorno de las evidencias para reorientar los errores en el transcurso de la asignatura.

Por otro lado, había convergencia en las opiniones de expertos, docentes y alumnos, en que resultaría más efectivo menos cantidad de evidencias, pero con más calidad, con trabajos integrados y con un sentido nuclear en la carrera universitaria.

Otra cuestión importante del análisis que realizamos se centró en los retornos de las evidencias y el feedback que debería producirse durante el proceso de aprendizaje. En el grupo de discusión de docentes se incidía en que había que encontrar el modo de facilitar el aprendizaje y la manera de hacerlo era a través del feedback. Esto permitiría que el estudiante se situara en el proceso de aprendizaje además de permitir la corrección de errores in situ.

Al preguntarle a los alumnos si había un retorno de las evidencias, casi todos decían que generalmente no, que no tenían la posibilidad de saber qué es lo que habían hecho mal, que pasaban muchos días y semanas hasta saber la nota. Valorarían como positivo un retorno de las evidencias para mejorar su aprendizaje, pero que muy pocos profesores les ofrecían este retorno. En palabras de una alumna del grupo de discusión de cuarto curso: no hay retorno, o sea, hay un retorno cuando ya tienes la nota final por si quieres saber en qué has fallado, pero ya no lo puedes mejorar. Una docente apuntaba en el grupo de discusión que si no tienes la posibilidad de hacer retorno y ya le estás pidiendo la segunda evidencia, acaba siendo contradictorio hasta para el mismo profesor.

Los datos que extrajimos fueron, que el 60 por ciento de alumnos pensaba que casi nunca o nunca eran retornadas las evidencias y percibían el no retorno como un sistema poco favorecedor en el aprendizaje, ya que no creían que se produjera esta reorientación.

Por otro lado, los alumnos valoraban muy positivamente que se produjera este retorno (con una valoración media de un 8,4 sobre 10) y lo consideraban una herramienta muy útil para reorientar los errores y mejorar en su proceso de aprendizaje.

La realidad expresada en relación a las tutorías fue la siguiente:

El 53 por ciento de alumnos afirmaba no realizar nunca o casi nunca tutorías durante el proceso de elaboración de las evidencias, frente al 42 por ciento que decía que algunas veces y el 5 por ciento que manifestaban realizarla casi siempre.

También los alumnos destacaban la importancia y la utilidad de la tutorización individualizada. Como inconvenientes resaltan que debido a la poca disponibilidad del profesorado y el gran número de alumnos por aula, la tutorización individualizada es algo que no se lleva a cabo de forma óptima.

En el grupo de discusión de docentes también se apunta que el tamaño del grupo es un

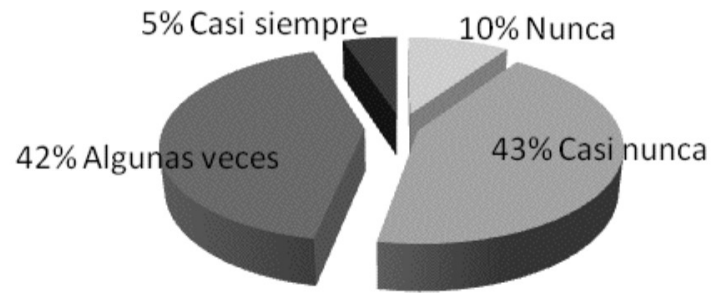

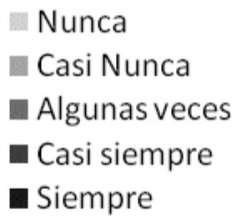

Nunca

Casi Nunca

- Casi siempre

Siempre

Gráfico 2. Realización de tutorías en el proceso de elaboración de las evidencias. 
factor que influye directamente en la posibilidad de hacer una tutorización individualizada.

Respecto a la necesidad de superar la asignatura con un examen final, el $88 \%$ de los alumnos creen que la realización de un examen y la necesidad de aprobarlo no entra dentro de evaluación continuada. Delgado y Oliver (2006) afirman que es importante tener en cuenta que si la evaluación continua está bien diseñada, las asignaturas se deberían poder superar sin necesidad de realizar una prueba de evaluación final o como mínimo la prueba final de evaluación debería ser diferente para los alumnos con evaluación continua en comparación con aquellos que no la han elegido. El profesor no sólo debe evaluar al final del proceso de aprendizaje la asimilación de conocimientos y el desarrollo de competencias por parte de los estudiantes, sino que, a lo largo del curso, debe proponer con cierta periodicidad actividades, de carácter evaluable, que faciliten la asimilación y el desarrollo progresivos de los contenidos de la materia y de las competencias que deben alcanzarse, respectivamente. De esta forma, la evaluación se convierte en continua o progresiva, y el profesor puede realizar un mayor y mejor seguimiento del progreso en el aprendizaje del estudiante.

A la hora de planificar las actividades de evaluación, el docente debe efectuar el cálculo del esfuerzo teniendo en cuenta que cada estudiante tiene su propio ritmo de trabajo y de aprendizaje. Según Delgado y Oliver como mínimo el punto de partida para determinar el esfuerzo medio del estudiante se encuentra en el cálculo del tiempo y la dificultad que supone para el propio profesor la resolución de la acti-
vidad.Además, es necesario diseñar éstas actividades de evaluación continua de forma flexible, de manera que los estudiantes puedan adaptarse individualmente a las mismas.

\subsection{Implementación}

Cuando la cuestión de la implementación de la evaluación continua se plantea a los alumnos el resultado de los cuestionarios es contundente, el 90 por ciento de alumnos creen que es regular, deficiente o muy deficiente la implementación de la evaluación continua.

En los grupos de discusión de los alumnos, algunos manifiestan aspectos negativos referentes a la implementación y no creen que haya mejorado la calidad de la enseñanza, aunque tenemos que destacar que estos no pueden compararlo con la calidad de la enseñanza en la diplomatura de Trabajo Social, porque no formaron parte de ella.

A pesar de esta valoración existe la percepción de un 77 por ciento entre los alumnos, de que los conocimientos adquiridos durante el Grado de Trabajo Social son adecuados y los podrán utilizar al acabar el grado.

Los resultados sobre la percepción de los alumnos respecto a la cuestión de si en la Universidad existen los recursos necesarios para llevar a cabo una buena docencia, es poco o nada positivo.

Valoran que los grupos están masificados y afirman que faltan horas de tutorías. También se quejan de la calidad y cantidad del personal docente y de la falta de personal y horarios de Secretaría. Asimismo, consideran que tienen a su disposición pocos ordenadores y que la conexión con internet es demasiado lenta. Los medios materiales son a su entender malos, las si-

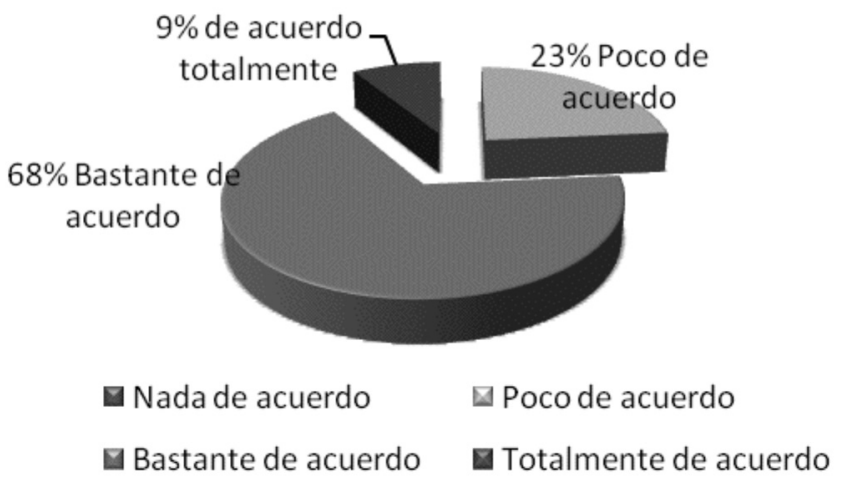

Gráfico 3. Aplicación de los aprendizajes y conocimientos al finalizar el Grado de Trabajo Social. 
llas incómodas, las aulas pequeñas y con malas condiciones acústicas. La nota que le asignan los alumnos a los recursos es de un 5,2.

La falta de recursos e instrumentos adecuados provoca que Mateo plantee la siguiente cuestión: "¿Cómo vas a hacer funcionar un planteamiento como el de Bolonia con las estructuras que tenemos? Si este es el problema [...] a Bolonia le ha pasado lo que le paso en su día a la LOGSE que se aprueba la ley en época de vacas gordas, y después se desarrolla en época de vacas flacas».

Por otro lado, algunos alumnos participantes en los grupos de discusión de alumnos verbalizan que existe un peligro real de infantilizar la educación universitaria (lo comparan con la E.S.O. y manifiestan que la asistencia a clases está sobrevalorada).

Los expertos y docentes entrevistados opinan que la implementación no se está llevando a cabo correctamente. Inciden en que hay una serie de factores que no ayudan en su desarrollo y señalan la necesidad formación y asesoramiento del profesorado en la implementación de la evaluación continua. En este sentido encontramos consenso en las diferentes opiniones de expertos y docentes que afirmaron que la aplicación de esta metodología encuentra resistencias producidas por la masificación de las aulas tal como afirmaban los alumnos, destacando que las clases no suelen bajar de los 60 alumnos (en la mayoría de las asignaturas). Esta situación hace que la mayoría de universidades no estén preparadas para afrontar el reto que la implementación de la evaluación continua supone. Ante esta situación de masificación, Mateo explica que una posible solución pasaría por programar dos o tres actividades nucleares, y el resto, conformarnos con un tipo de enseñanza más acorde con la realidad. Báñez, profesora de Grado de Trabajo Social de la UB, quien aportaba su experiencia como jefe de estudios del Grado de Trabajo Social en la Universidad de Zaragoza, destacaba que la evaluación continua aplicada en sentido estricto requiere un contexto educativo y unas condiciones que en la Universidad española, actualmente, no se dan.

En relación a la calidad de la enseñanza, la decana de la Facultad de Pedagogía, opina que la calidad ha mejorado en cuanto a que el aprendizaje del estudiante está más ajustado a lo que tendrá que desarrollar en su vida laboral. La implicación y motivación de los profesores es indispensable para una buena implementación de la evaluación continua. La impresión de la directora del Departamento de Trabajo Social y Servicios Sociales de la UB, es que también la calidad ha mejorado, aunque falta aún comparar los resultados que se obtenían antes de su implementación con los actuales. Esto contrasta con la opinión manifestada por el alumnado a través de los cuestionarios, donde el 70 por ciento está poco o nada de acuerdo con que la calidad de la enseñanza impartida haya mejorado.

Por su parte, el Jefe de estudios del Grado de Trabajo Social, manifiesta que las medidas que implican una reducción del número de profesores afectan de manera clara a la implantación del sistema, que reclama una plantilla estable de docentes. La directora del Grado de Trabajo Social, por su parte reconoce que hoy por hoy no podemos plantearnos escenarios óptimos. Hay consenso de opinión en relación a que el profesorado asociado esta poco reconocido tanto a nivel salarial, en condiciones de trabajo y en el reconocimiento como investigadores.

Mateo explica que «se están substituyendo profesores de dedicación completa por profesores asociados». Además expresa que «igual que ha habido un desequilibrio en el número de alumnos se ha producido un desequilibrio en la estructura del profesorado». Respecto a los profesores asociados, opina que se les paga estrictamente para hacer sus horas de clase, ¿Cómo le vas a pedir a una persona que le pagan $400 \mathrm{eu}$ ros que se reúna, que haga trabajos, actividades, que hable con los alumnos...? ¿Cómo se lo vas a pedir?

Los datos de la Facultad de Pedagogía de la Universidad de Barcelona reflejan que en Septiembre del 2012 del total de los 50 profesores del Departamento de Trabajo Social, 29 de ellos eran profesores asociados, es decir, el 58 por ciento del profesorado del Departamento de Trabajo Social. Este hecho es bastante común en muchos de los departamentos de la Universidad de Barcelona.

Ante las diferencias contractuales y la nueva situación en que se encuentra el contexto universitario, la directora del Departamento de Trabajo Social, opina que «el profesorado asociado tiene mucha más docencia y menos posibilidades de dedicarse a otras labores» y añade que a los profesores asociados no se les puede pedir lo 
mismo ya que la sobrecarga no está reconocida por la universidad.

Por otra parte, existe la percepción mayoritaria del aumento de trabajo tanto por parte de profesores como por parte de alumnos. A los mismos docentes les resulta contradictorio esta continua demanda y exigencia de evidencias a los estudiantes, si el mismo profesor por tiempo y por número de alumnos no es capaz de ir realizando una devolución de dichas evidencias. Báñez manifestaba que puesto que no existe tiempo de hacer un seguimiento y devolución de forma continua «es mejor que la evaluación sea menos continua pero que sea más real, en cuanto a lo que el profesor pide, y que lo pueda corregir y devolver».

En las entrevistas exploratorias realizadas a expertos que aportan otras experiencias de la evaluación continua, Giménez, profesor titular de la Facultad de Física y Química de la UB, considera que hay una cierta resistencia por parte del profesorado puesto que el esfuerzo y el trabajo es mucho mayor, ya que las tareas del profesorado no se dedican tan solo al tiempo de corrección sino además al tiempo de preparación del material y de seguimiento de los alumnos.

\section{Conclusión}

El término «evaluación continua» se presta a confusión y la realidad analizada nos indicó que los docentes realizan pruebas periódicas a lo largo de todo el periodo lectivo que finalmente serán sumadas y ponderadas, en ningún momento se tiene en cuenta que el objetivo principal es perfeccionar el proceso de formación del alumno: «aprender a aprender». Son pruebas calificativas y no formadoras. Suscriben esta afirmación casi todos los docentes a los que se le realizado una entrevista.

En cuanto al alumnado todo y que el 70 por ciento de los alumnos creían tener un alto conocimiento de lo que representa la evaluación continua, comprobamos que desconocían qué significaba la evaluación continua y la identificaban con la realización sistemática y constante de pruebas evaluativas sumatorias.

Joan Mateo afirmaba que se debían realizar actividades evaluativas de principio a fin combinadas con clases magistrales en las que se produjera una participación más activa del alumno. No obstante, los docentes y expertos consultados reconocían no tener unas directrices claras y delimitadas en relación a la metodología que se circunscribiría dentro de la implementación del nuevo plan educativo, Bolonia.

Después de realizar una revisión de los planes docentes se llegó a la conclusión que las evidencias que se proponen, no seguían un criterio unificado, ni en la cantidad ni en la proporción porcentual en los ítems evaluativos. Hecho que dificulta la adquisición de las competencias basadas en conocimientos, capacidades, habilidades y actitudes, que caracterizan la hoja de ruta académica propuesta por el nuevo paradigma educativo.

Por otro lado, tampoco se ajustaban a las necesidades expresadas por los alumnos ni a las exigencias especificadas para cada asignatura, y lo que es más importante, en los planes docentes analizados no aparecía el compromiso de retorno por parte del profesor con la consiguiente posibilidad de mejora.

De la investigación se extrae que:

- La masificación en las aulas impide la proximidad entre profesor y alumnos que permita un seguimiento eficaz.

- Existe una percepción generalizada de que los recursos materiales son insuficientes.

- No se dedican los suficientes medios para la formación del profesorado que permita la unificación de criterios en relación a la implementación continuada así como resulta escasa la coordinación interdepartamental que cohesione dichos criterios. Así como, debemos destacar la incidencia que tiene en la calidad, la situación laboral de muchos profesores asociados que han visto cierta precarización en sus condiciones laborales.

- Hay una tasa más elevada de aprobados entre los que eligen evaluación continua, según el 80 por ciento de alumnos, mientras que los docentes rebaten afirmando que esta circunstancia no debería producirse.

- No existe un compromiso de retorno y no se realizan las suficientes tutorías que permitan a los alumnos rectificar en su proceso de aprendizaje. Lo cual acaba incidiendo en que existe una opinión desfavorable por parte del alumnado en cuanto a la calidad de la enseñanza.

- Que existe un reconocimiento explícito por parte de la mayoría de docentes de que ni la calidad ni la cantidad de evidencias es la que debería, ya que en algunas ocasiones se llegan a duplicar contenidos produciéndose así una so- 
brecarga de trabajo, tanto en docentes como en alumnos.

- Se hace evidente que de alguna manera la implementación de la evaluación continuada excluye a las personas que alternan la vida académica con la vida laboral.

Finalmente, debemos señalar que encontramos una opinión casi unánime sobre el proceso de infantilización que se había producido en la universidad a partir de la implementación del plan Bolonia, siendo un porcentaje muy bajo el que opinaba que se habían producido ciertas mejoras, como una mayor responsabilidad del alumnado, dentro y fuera de las aulas, así como un ajuste de los aprendizajes a la vida real.

Los datos hasta aquí expuestos muestran que la normativa impuesta por la Universidad de Barcelona en el Grado de Trabajo Social, responde a criterios burocráticos sin considerar las necesidades reales, o haber contemplado y analizado la realidad que viven docentes y alumnos. Por otro lado, cabe destacar que en lo que respecta a la mejora de la calidad de la enseñanza nos faltaron datos comparativos con la calidad de la enseñanza que se ofrecía en la extinguida diplomatura para poder valorar de forma más global «la mejora».

Concluimos pues, que lo fundamental es entender que sin retorno, sin feed back entre profesor y alumno, no existe evaluación continua, podrá ser una evaluación sumativa, donde el alumno presentará diferentes evidencias que serán calificadas y ponderadas con una evaluación acreditativa, pero esto no es la evaluación continua. En la evaluación continua está implícita la evaluación formativa donde existe posibilidad de mejora, de rectificación y de reflexión que incida en un verdadero proceso de aprendizaje. Si no hay retorno no hay evaluación continua. Lo cual resulta inviable, según la opinión generalizada de los docentes, si no se produce un aumento en los recursos humanos y técnicos, la adecuación e interconexión de espacios, que dadas las circunstancias socio-económicas por las que atravesamos parece inviable e incluso, por qué no decirlo, utópico.

A pesar de todo lo expuesto, existe la percepción de más de tres cuartas partes de los alumnos, de que los conocimientos adquiridos durante el Grado de Trabajo Social son adecuados y los podrán utilizar al acabar el grado.

\section{Referencias bibliográficas}

Coll Salvador, C., Mayordomo Saiz, RM., Naranjo Llanos, M. y RocheraVillach, M. J. (2007). Evaluación continua y ayuda al aprendizaje. Análisis de una experiencia de innovación en educación superior con apoyo de las TIC. Revista electrónica de investigación psicoeducti$v a, 5(13)$.

Comisió Acadèmica del Consell de Govern. (2008). Glosari acadèmic y docent de la Universitat de Barcelona. Universitat de Barcelona.

Delgado, A. y Oliver, R. (2006). La evaluación continua en un nuevo escenario docente. Revista de Universidad y Sociedad del Conocimiento, 3(1) [on line].

Escofet, A., Martínez, F. y Mateo, J. (2009). Naturaleza del cambio en la concepción pedagógica del proceso de enseñanza-aprendizaje en el marco del EESSS. Una experiencia para el análisis. Revista Fuentes, 9, 53-96.

García Martínez, J. (2009). La ordenación académica ante los desafíos del Espacio Europeo de Educación Superior. Revista Fuentes, 9, 42-52.

Gallardo, E. y Montolio, D. (2011). ¿Existe relación entre la evaluación continua y los resultados de los alumnos? E-pública: revista electrónica sobre la enseñanza de la economía pública, 8, 6379. Consultada en 15/3/2012 en www.unizar.es/e-publica

Imbernon, F. y Medina, J. L. (2008). Metodología participativa en el aula universitaria. La participación del alumnado. Cuadernos de docencia universitaria. ICE y Editorial Octaedro.

Lima, A. (2011). Diez preguntas sobre grado. La implantación del Grado en Trabajo Social en las Universidades. Servicios Sociales y Politica Social, 96, 75-88.

Mateo, J. y Vlachopoulos, D. (2010). La nueva naturaleza del aprendizaje y de la evaluación en el contexto del desarrollo competencial, retos europeos en la educación del siglo XXI. Revista iberoamericana de evaluación educativa, 3(3). 
Ministerio de Educación Cultura y Deporte. (2003). La integración del sistema universitario español en el Espacio Europeo de enseñanza superior. Documento-marco. Madrid: Ministerio de Educación, Cultura y Deporte.

Sursock, A. y Smidt, H. (2010). Trends 2010: A decade of change in European Higher Education. EEES. Obtenido en http://eees.universia.es/documentos/asociacion-universidad-europea/. 